\title{
Fundus imaging in patients with cataract: role for a variable wavelength scanning laser ophthalmoscope
}

\author{
J N P Kirkpatrick, A Manivannan, A K Gupta, J Hipwell, J V Forrester, P F Sharp
}

\begin{abstract}
Aims-An investigation was carried out to compare the image quality of the ocular fundus obtained clinically, photographically, and with the scanning laser ophthalmoscope (SLO) at visible and infrared wavelengths in patients with significant cataract.

Methods-Nineteen patients admitted for routine cataract extraction were examined clinically by two independent observers to ascertain cataract type and clarity of fundus view with an indirect ophthalmoscope. Fundus photography and both confocal and direct (non-confocal) SLO imaging at $590 \mathrm{~nm}, 670 \mathrm{~nm}$, and $830 \mathrm{~nm}$ were carried out after pupillary dilatation. Images obtained were graded independently using a recognised grading system.

Results-Quality of SLO images appeared to be superior to indirect ophthalmoscopy $(p<0.01)$ and fundus photography $(\mathbf{p}<0.001)$ when graded subjectively. Quantitative analysis of contrast of retinal vessels demonstrated significantly higher contrast for the SLO compared with digitised fundus photographs at all wavelengths tested $(p<0 \cdot 001)$, with highest contrast at $590 \mathrm{~nm}$. Use of a confocal aperture significantly improved vessel contrast but may reduce overall image intensity.

Conclusions-Scanning laser ophthalmoscopy may offer a method to observe and record fine fundus detail in patients who have marked cataract.

(Br f Ophthalmol 1995; 79: 892-899)
\end{abstract}

University of

Aberdeen, Medical

School, Foresterhill,

Aberdeen AB9 2ZD

Department of Ophthalmology

J N P Kirkpatrick

A K Gupta

J V Forrester

Department of Biomedical Physics A Manivannan J Hipwell

P F Sharp Correspondence to: Department of

Ophthalmology, Royal Perth Hospital, GPO Box X2213, Perth 6001, Western Australia.

Accepted for publication 18 July 1995

Various methods are available to the ophthalmologist for examination of the fundus of the cataractous eye. Clinical methods such as indirect ophthalmoscopy are rapid and easy to use giving an acceptable image quality in patients with moderate cataract. A permanent record of the fundus may be achieved using fundus photography but results may be disappointing. The scanning laser ophthalmoscope (SLO) may offer an alternative to fundus photography in providing a high resolution digital image at a variety of incident wavelengths. It is an investigational tool which has allowed acquisition of real time fundus angiography, ${ }^{1-3}$ infrared ophthalmoscopy, ${ }^{4}$ and, more recently, retinal and optic disc tomography. ${ }^{56}$ Initial observations using the SLO improved detail in patients with significant cataract. ${ }^{4}$ The use of a monochromatic source to reduce chromatic aberration together with the reduced optical density of aging lens nuclei to longer wavelengths ${ }^{78}$ may result in improved image quality. In addition a fine, highly collimated laser beam might penetrate areas of the cataractous lens which are less dense and thereby reduce light scatter.

This paper investigates the ability of a multiwavelength confocal SLO to provide useful fundus images in patients with cataract. It compares the subjective assessment of image quality with images from clinical indirect ophthalmoscopy and fundus photography. In addition, quantitative analysis of the contrast of retinal vessels is compared between SLO images at different wavelengths and digitised fundus photographs.

\section{Patients and methods}

\section{PATIENT RECRUITMENT}

Approval was obtained from the joint ethics committee of the University of Aberdeen and Aberdeen Royal Hospitals NHS Trust for this study. From patients admitted for routine cataract surgery at Aberdeen Royal Infirmary, 19 eyes of 19 patients were selected for inclusion. Patients were excluded if there was mature cataract, significant media opacity other than cataract, or if the pupil did not dilate to greater than $4 \mathrm{~mm}$. All patients gave written informed consent.

\section{CLINICAL EXAMINATION}

After pupillary dilatation with tropicamide $1 \%$ and phenylephrine $2.5 \%$ patients were have suggested that this instrument may offer

examined by slit-lamp biomicroscopy and in direct ophthalmoscopy by two independent experienced ophthalmologists (JNPK and AKG).

The presence of cortical, nuclear, and posterior subcapsular opacities was classified according to the LOCS II system by comparison with a set of standard photographs. ${ }^{9}$ In this technique cortical cataract is graded on a scale from 1 to 4 , nuclear colour on a scale of 1 to 3 , nuclear opacity on a scale of 1 to 3 , and posterior subcapsular cataract on a scale of 1 to 3 . For the purposes of the present study the nuclear cataract grading was estimated by degree of opacity alone and nuclear colour was not used for subsequent analysis.

The clarity of detail seen at the posterior pole with indirect ophthalmoscopy was 
Table 1 Grading system for optic disc image clarity (adapted from Nussenblatt et $\mathrm{al}^{10}$ )

\begin{tabular}{ll}
\hline $\begin{array}{l}\text { Grading } \\
\text { score }\end{array}$ & Optic disc features \\
\hline 0 & $\begin{array}{l}\text { Fundus detail clear } \\
\text { Slight blurring of optic disc margin and fine vessels }\end{array}$ \\
2 & $\begin{array}{l}\text { Fine vessels on optic disc visible but blurred } \\
\text { Only large disc vessels discernible. Disc margin } \\
\text { blurred } \\
\text { Optic disc margins just discernible. Major vessels } \\
\text { not seen }\end{array}$ \\
4 & Red reflux present but optic disc not seen \\
\hline
\end{tabular}

estimated using a modification of a scoring system developed for grading of severity of vitreous opacities in posterior uveitis. ${ }^{10}$ In particular the observers were concerned with the appearance and clarity of the optic disc and adjacent vessels (Table 1). Observers' results of clinical examination, including grading of optic disc clarity, were noted independently and no discussion between observers regarding their clinical assessments took place.

\section{IMAGING PHOTOGRAPHY}

Fundus photography and scanning laser ophthalmoscopy were carried out at the same sitting within a few minutes of one another. From each patient a minimum of four fundus photographs was taken by one operator (JNPK) using a Nikon Retinapan-45 fundus camera using Fujichrome 100 film with the flash setting at maximum intensity and 1/60 second exposure. A 35 degree field centred on the optic disc was used. Colour transparencies were graded independently by both observers by viewing on a light box with a $\times 10$ loupe. The same grading system was used as for indirect ophthalmoscopy (Table 1).

For quantitative analysis the transparency which was adjudged to be of best image quality for each patient was digitised by projection through a $550 \mathrm{~nm}$ interference filter using a highly collimated light source and a 25 degree field of the frame centred on the optic disc was focused onto an optically aligned, monochrome Kodak MegaPlus charge coupled device (CCD) camera ( $1320 \times 1024$ elements). Digital output from the camera was fed to the frame grabber of a Series 151 image processing system (Imaging Technology Inc, USA) permitting capture of each frame at a resolution of $1024 \times 1024$ pixels. The grey level for each grabbed transparency was adjusted so that the maximum intensity within the image was set at a level of 255 . The system was controlled by a SUN IPX workstation (Sun Microsystems, USA) through the VISILOG image processing software (Noesis, France).

\section{IMAGING - SCANNING LASER}

OPHTHALMOSCOPY

Scanning laser ophthalmoscopy was carried out using the custom built Aberdeen scanning laser ophthalmoscope. ${ }^{1112}$ This instrument is similar to commercially available instruments but permits a greater degree of flexibility with respect to confocal aperture size and wavelength source. All images were of a 25 degree field centred on the optic disc and consisted of $740 \times 511$ pixels. Patients were imaged at $830 \mathrm{~nm}, 670 \mathrm{~nm}$, and $590 \mathrm{~nm}$ using diode laser sources for $830 \mathrm{~nm}$ and $670 \mathrm{~nm}$ while a tunable rhodamine-6-G dye laser (Spectra Physics-375, USA), pumped by a 1 W argon laser (Spectra Physics Stabilitic-2017, USA) was used for the $590 \mathrm{~nm}$ image. The entrance and exit pupil sizes of this SLO were $1 \mathrm{~mm}$ and $6 \mathrm{~mm}$ in diameter respectively and the incident power at the cornea for all laser sources was set at $200 \mu \mathrm{W} / \mathrm{cm}^{2}$. Patients were imaged both with and without a confocal aperture of $400 \mu \mathrm{m}$ diameter and the gain control for the detector was adjusted to provide an image centred on the optic disc with the optimum contrast. A minimum of two images for each aperture size and wavelength setting were grabbed and transferred to the SUN IPX workstation for subsequent grading (Table 1) and image analysis.

\section{IMAGE PROCESSING AND QUANTITATIVE} ANALYSIS

For convenience of image handling and storage digitised fundus photographic images were reduced to $512 \times 512$ pixels. SLO images were converted to a similar format by cutting unwanted pixels from the sides of the images and adding a row of pixels of zero value to the top of the image. Despite attempts to register the images to allow automated processing the image quality in many of the images was poor and both automated and manual registration techniques were considered impractical.

A quantitative comparison of digitised fundus photographs and SLO images was carried out as follows. From the available images of each patient the best quality images from fundus photography, and SLO imaging at $830 \mathrm{~nm}, 670 \mathrm{~nm}$, and $590 \mathrm{~nm}$, with and without the confocal aperture, were selected for processing. Using binary image drawing tools within the software program it was possible to create a series of binary 'mask' images which could select out a small portion of the original image for analysis. For each image a first order branch retinal arteriole and venule with an immediately adjacent area of background fundus close to the optic disc was selected. Three separate masks of approximately 1000 pixels in area were created to overlap arteriole, venule, and background respectively. For each patient studied the same portion of arteriole, venule, and background fundus was selected manually from all of the images, both photographic and SLO images, and subjected to quantitative analysis. A typical SLO image with overlying image masks is shown in Figure 1. The statistical data concerning the grey levels of the pixels in the original image covered by each mask could then be calculated and transferred to a spreadsheet. The data recorded were the number of pixels, minimum and maximum grey levels, mean grey level, and standard deviation of the grey levels. Using these data a simple measure of image quality, object contrast, could be defined as follows: 


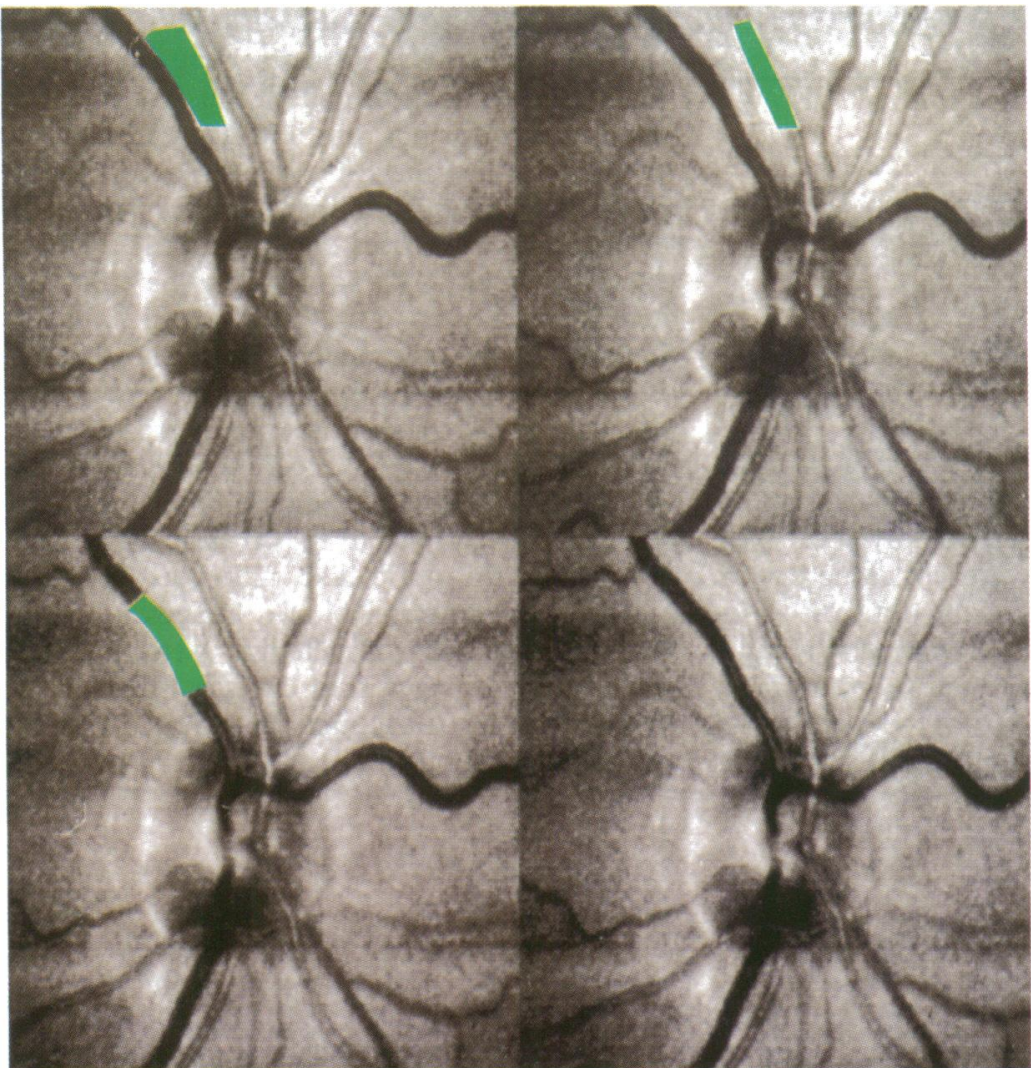

Figure 1 Four scanning laser ophthalmoscope images of optic disc imaged at $590 \mathrm{~nm}$ with confocal aperture. Overlying areas marked in green are the binary masks used to compare contrast of background fundus reflectance (top left) with adjacent arteriole (top right) and venule (bottom left). Note image noise accentuated by photographic reproduction.

Arteriole contrast $=\frac{\text { Mean grey level below 'background mask' }}{\text { Mean grey level below 'arteriole mask' }}$

and

Venule contrast $=\frac{\text { Mean grey level below 'background mask' }}{\text { Mean grey level below 'venule mask' }}$

where a value of 1 implies no contrast - that is, no discrimination between object and background.

In addition, the ability to discriminate between two structures depends not only on the ratio of mean grey level but also on the noise or random variation in the two structures being compared. Using data concerning variance of pixel distributions, $95 \%$ confidence limits for each mask area were calculated to determine whether the two adjacent regions were significantly different in grey level characteristics.

\section{STATISTICAL ANALYSIS}

Interobserver variability for grading of cataract type was analysed by use of a weighted kappa (K) score. Interobserver variation for the grading of image quality of indirect ophthalmoscopy, fundus photography, and SLO imaging was also calculated. Intraobserver variation for grading of image quality was carried out on all of the SLO images arranged in random order 4 weeks after the initial grading process.

For subsequent comparison of subjective grading between image groups the mean observer score from the two observers was used and groups were compared using a non-parametric Wilcoxon matched pairs analysis.
Correlation between cataract type grading and subjective image quality scores was analysed using Kendall's rank correlation.

Comparison between arteriole and background 'masks' and between venule and background 'masks' for individual images was carried out using $5 \%$ and $95 \%$ confidence limits as these image regions were assumed to have a normal distribution. For comparison of contrast ratios between digitised fundus photographs and SLO images the Wilcoxon matched pairs test was applied.

For all statistical tests significance was assumed if the value of $p$ was less than $0 \cdot 05$.

\section{Results}

QUALITATIVE DATA

The mean age of the patients included in the study was 76.3 (SD 9.1) years and there were four males and 15 females. Details of the cataract type grading according to the LOCS II system are shown in Table 2 . Weighted kappa scores for interobserver variability of grading of cortical cataract, nuclear opacity, and posterior subcapsular cataract were $0.84,0.76$, and 0.83 respectively, showing good agreement between observers.

From the 19 patients included in the study at the time of clinical examination, 17 underwent satisfactory fundus photography with a camera failure leading to loss of fundus photographs in two patients (patient numbers 17 and 19). All patients underwent SLO imaging but attempts were not successful in all patients at all wavelengths. In one patient (patient number 8) non-confocal images at all three wavelengths were not recorded. This patient's data were excluded from any comparison of confocal and non-confocal imaging. For SLO imaging in general, it was more difficult to achieve a recordable image with confocal imaging compared with non-confocal techniques and, furthermore, imaging at $590 \mathrm{~nm}$ and $670 \mathrm{~nm}$ was found to be more difficult than at $830 \mathrm{~nm}$. For this reason the SLO image series is not complete for patient numbers, $4,7,12$, 13, 15, 16, and 17 (see Table 3).

Table 2 Grading of cataract type for 19 patients according to LOCS II system ${ }^{9}$

\begin{tabular}{llll}
\hline \multicolumn{5}{c}{$\begin{array}{l}\text { Image grading score } \\
\text { Patient }\end{array}$} & $\begin{array}{l}\text { Cortical } \\
\text { No }\end{array}$ & $\begin{array}{l}\text { Nuclear } \\
\text { (grade 0-4) }\end{array}$ & $\begin{array}{l}\text { Posterior subcapsular } \\
\text { (grade 0-3) }\end{array}$ \\
\hline 1 & 3 & 1 & 2 \\
2 & 3 & 3 & 3 \\
3 & $1 \cdot 5$ & 1 & 1 \\
4 & $2 \cdot 5$ & 1 & 2 \\
5 & $2 \cdot 5$ & 3 & $0 \cdot 5$ \\
6 & $2 \cdot 5$ & 1 & 3 \\
7 & 4 & 3 & 0 \\
8 & 3 & 2 & $2 \cdot 5$ \\
9 & 1 & $2 \cdot 5$ & 3 \\
10 & 1 & 3 & 2 \\
11 & 1 & 2 & 3 \\
12 & 2 & 3 & 3 \\
13 & 1 & 3 & 3 \\
14 & 1 & $2 \cdot 5$ & $0 \cdot 5$ \\
15 & 3 & 3 & 0 \\
16 & 1 & $2 \cdot 5$ & 3 \\
17 & 4 & 2 & 3 \\
18 & 3 & 3 & 3 \\
19 & 1 & $1 \cdot 5$ & $0 \cdot 5$ \\
\hline
\end{tabular}


Table 3 Mean image grading score for 19 patients from clinical examination, fundus photography, and scanning laser ophthalmoscope imaging at 590, 670, and $830 \mathrm{~nm}$ with and without confocal aperture

\begin{tabular}{|c|c|c|c|c|c|c|c|c|}
\hline \multirow{3}{*}{$\begin{array}{l}\text { Patient } \\
\text { No }\end{array}$} & \multirow[b]{3}{*}{ Clinical } & \multirow[b]{3}{*}{ Photo } & \multicolumn{6}{|c|}{ Scanning laser ophthalmoscopy } \\
\hline & & & \multicolumn{2}{|l|}{$590 \mathrm{~nm}$} & \multicolumn{2}{|l|}{$670 \mathrm{~nm}$} & \multicolumn{2}{|l|}{$830 \mathrm{~nm}$} \\
\hline & & & Confocal & Direct & Confocal & Direct & Confocal & Direct \\
\hline 1 & 1.5 & 1 & 1 & 2 & 2 & 2 & 2 & 2 \\
\hline 2 & 2 & 3 & 2 & 2 & 2 & 2 & 2 & $2 \cdot 5$ \\
\hline 3 & 2 & 2.5 & 2 & 2 & 2 & 3 & 2 & 2 \\
\hline 4 & 3 & 3 & NR & 1.5 & NRR & 3 & NR & 3 \\
\hline 5 & 1 & 3 & 3 & 1.5 & 2 & 2 & 1.5 & 2 \\
\hline 6 & 3 & 3.5 & 3 & 2 & 2.5 & 2 & 2 & 2 \\
\hline 7 & 4 & 5 & NR & NR & NR & NR & NR & 5 \\
\hline 8 & 1 & 1.5 & 2 & $\star$ & 2 & $\star$ & 2 & $\star$ \\
\hline 9 & 1.5 & 2 & 2 & 1.5 & 2 & 2 & 2 & 2 \\
\hline 10 & 2 & 3 & 2 & 2 & 2 & 3 & 2 & 3 \\
\hline 11 & 1.5 & 2 & 1 & 1 & 1.5 & 1.5 & 2 & 2 \\
\hline 12 & 4 & 4 & NR & 3 & NR & 4 & NR & $2 \cdot 5$ \\
\hline 13 & 4 & 4 & $\mathrm{NR}$ & 2 & NR & 3 & 2 & 3 \\
\hline 14 & 1 & $2 \cdot 5$ & 1 & 1 & 1.5 & 1.5 & 1 & 1.5 \\
\hline 15 & 4 & 4 & NR & NR & NR & 3 & NR & 3 \\
\hline 16 & 3 & 3 & NR & 2 & $2 \cdot 5$ & 2 & 2 & 2 \\
\hline 17 & 3 & * & NR & 5 & 2.5 & $2 \cdot 5$ & 1.5 & 2 \\
\hline 18 & 4 & 3 & 2 & 1 & 2 & 2 & 1.5 & 2 \\
\hline 19 & 1 & 夫 & 3.5 & 1 & $\overline{2}$ & 1 & 1.5 & 2 \\
\hline
\end{tabular}

$\mathrm{NR}=$ image not recordable; ${ }^{\star}=$ image recordable but not stored.

Mean values for clarity of optic disc detail graded by the two observers for indirect ophthalmoscopy, fundus photography, and SLO imaging with and without confocal aperture at $590 \mathrm{~nm}, 670 \mathrm{~nm}$, and $830 \mathrm{~nm}$ are shown in Table 3. For fundus photography the grading score is applied to the best of four images while for SLO images the grading score is that for the better of two images at each wavelength, with and without confocal aperture. Interobserver variation for indirect ophthalmoscopy, fundus photograph, and SLO image grading was calculated to be $0.82,0.77$, and 0.55 respectively which shows an acceptable level of agreement. Intraobserver variation for observer one for SLO images was found to be 0.62 which also shows a good degree of reproducibility.

Taking the SLO images for each patient as a group the lowest grading score (best quality) for any image is shown in Table 4 and compared with the scores for indirect ophthalmoscopy and fundus photography taken from Table 3. In general the quality of fundus

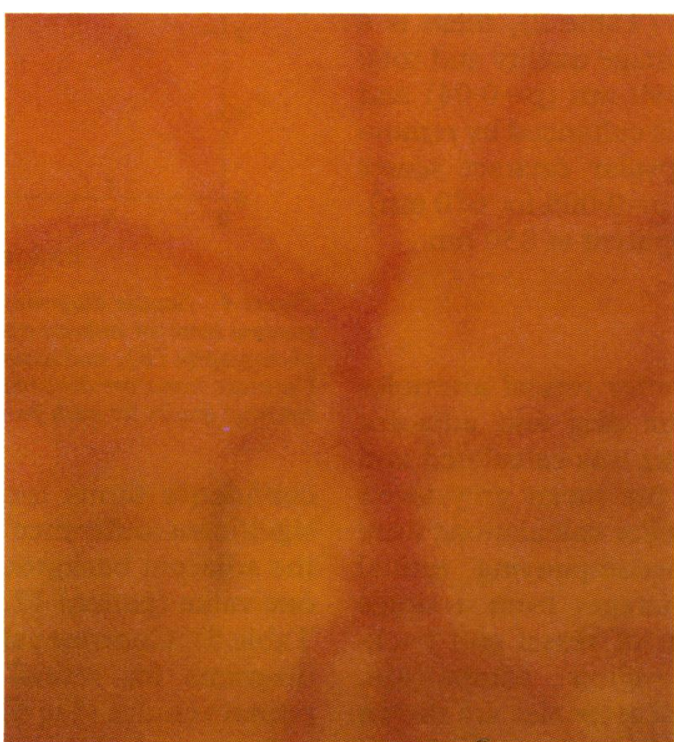

Figure 2 Comparison of colour fundus photograph of optic disc (left) and scanning laser ophthalmoscope (SLO) image at $590 \mathrm{~nm}$ (right) with confocal aperture. Both images have been reduced to approximately 10 degree field. Contrast of vessels and optic cup is greater for SLO image.

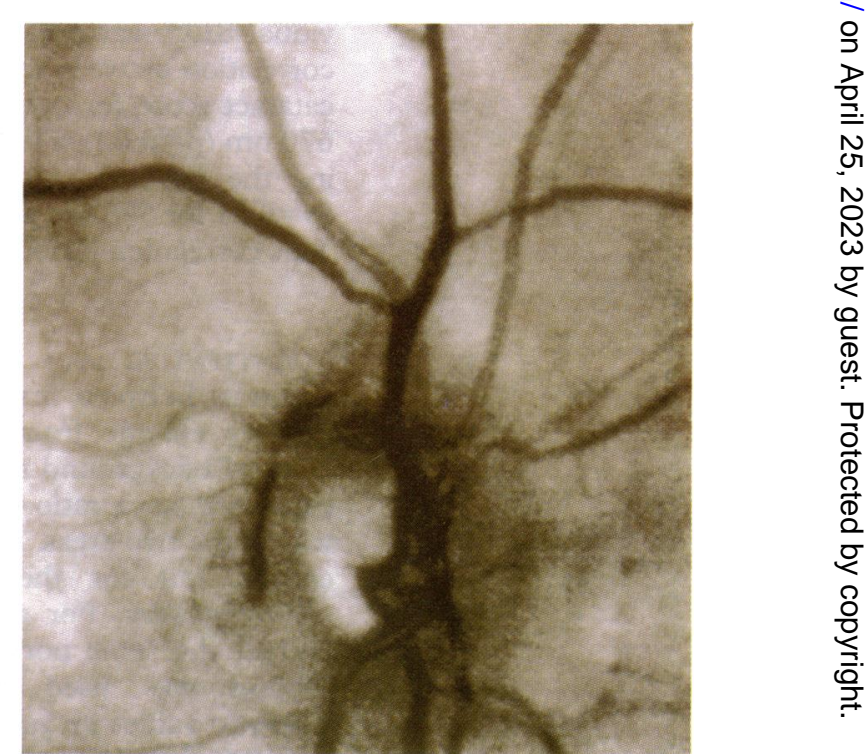

Table 4 Summary of image grading score for 19 patients with optimum scanning laser ophthalmoscopy (SLO) imaging wavelength recorded

\begin{tabular}{cllll}
\hline & \multicolumn{3}{c}{ Image quality grading score } \\
\cline { 2 - 3 } $\begin{array}{c}\text { Patient } \\
\text { No }\end{array}$ & Clinical & Photo & $\begin{array}{l}\text { Optimum } \\
\text { SLO }\end{array}$ & $\begin{array}{l}\text { Wavelength details of } \\
\text { optimum SLO image }\end{array}$ \\
\hline 1 & $1 \cdot 5$ & 1 & 1 & $590 \mathrm{c}$ \\
2 & 2 & 3 & 2 & $590 \mathrm{c}+\mathrm{d}, 670 \mathrm{c}+\mathrm{d}, 830 \mathrm{c}$ \\
3 & 2 & $2 \cdot 5$ & 2 & $590 \mathrm{c}+\mathrm{d}, 670 \mathrm{c}, 830 \mathrm{c}+\mathrm{d}$ \\
4 & 3 & 3 & $1 \cdot 5$ & $590 \mathrm{~d}$ \\
5 & 1 & 3 & $1 \cdot 5$ & $590 \mathrm{~d}, 830 \mathrm{c}$ \\
6 & 3 & $3 \cdot 5$ & 2 & $590 \mathrm{~d}, 670 \mathrm{~d}, 830 \mathrm{c}+\mathrm{d}$ \\
7 & 4 & 5 & 5 & N/A \\
8 & 1 & $1 \cdot 5$ & $1 \cdot 5$ & $830 \mathrm{~d}$ \\
9 & $1 \cdot 5$ & 2 & $1 \cdot 5$ & $590 \mathrm{~d}$ \\
10 & 2 & 3 & 2 & $590 \mathrm{c}+\mathrm{d} .670 \mathrm{c}, 830 \mathrm{c}$ \\
11 & $1 \cdot 5$ & 2 & 1 & $590 \mathrm{c}+\mathrm{d}$ \\
12 & 4 & 4 & $2 \cdot 5$ & $830 \mathrm{~d}$ \\
13 & 4 & 4 & 2 & $590 \mathrm{~d}, 830 \mathrm{c}$ \\
14 & 1 & $2 \cdot 5$ & 1 & $590 \mathrm{c}+\mathrm{d}, 830 \mathrm{c}$ \\
15 & 4 & 4 & 3 & $670 \mathrm{~d}, 830 \mathrm{~d}$ \\
16 & 3 & 3 & 2 & $590 \mathrm{~d}, 670 \mathrm{~d}, 830 \mathrm{c}+\mathrm{d}$ \\
17 & 3 & N/A & $1 \cdot 5$ & $830 \mathrm{c}$ \\
18 & 4 & 3 & 1 & $590 \mathrm{~d}$ \\
19 & 1 & N/A & 1 & $590 \mathrm{~d}, 670 \mathrm{~d}$ \\
\hline 'c'=confocal; 'd'= non-confocal. &
\end{tabular}

photographic detail is less than that seen with either the indirect ophthalmoscope or SLO. A typical example of a fundus photograph and SLO image (590 $\mathrm{nm}$ confocal) is shown in Figure 2. Using the Wilcoxon matched pairs test to compare groups, with the exclusion of data from patients 17 and 19 for photography, it is seen that SLO imaging shows a significant improvement over indirect ophthalmoscopy $(p<0.01)$ and both SLO imaging and indirect ophthalmoscopy are significantly superior to fundus photography $(\mathrm{p}<0.001$ and $\mathrm{p}<0.02$ respectively). Scatter diagrams represent these findings in Figure 3.

Table 4 also shows which SLO imaging technique resulted in the highest quality image for each patient. A number of patients showed similar optimum image quality at more than one wavelength (patient numbers $2,3,5,6,10$, $13,14,15,16$, and 19). For those 17 patients in whom it was possible to achieve images at $590 \mathrm{~nm}$ it was seen that this wavelength gave the highest optic disc score in 14. In the 


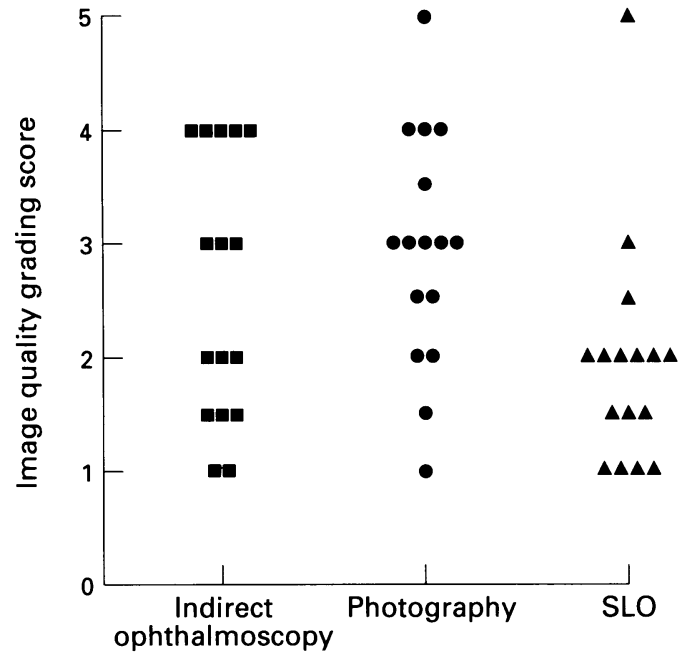

Figure 3 Scatter diagram showing qualitative grading scores for indirect ophthalmoscopy, fundus photography, and scanning laser ophthalmoscopy (SLO). Best image quality is given lowest grading score.

remaining five patients it was seen that imaging at $830 \mathrm{~nm}$ yielded best results in four patients while one patient had no useful SLO image data (patient 7). In no patient was $670 \mathrm{~nm}$ exclusively the optimum wavelength for fundus imaging.

Correlation of image quality with cataract type has been performed using Kendall's rank correlation coefficient, $\tau$. By adding the scores for cortical, nuclear, and posterior subcapsular cataract an overall cataract score with a possible maximum of 10 can be assigned to a given patient. Correlation of this score with image quality is significant for indirect ophthalmoscopy only $(p=0.025)$. If the score for posterior subcapsular cataract is not included in this total then both indirect ophthalmoscopy and fundus photography show significant correlation with the cataract grading score ( $p=0.03$ for both) (Fig 4). Quality of the optimum SLO image (Table 4) appears to show some correlation with nuclear cataract density $(p=0.04)$ but not with a combined cataract density score.

By looking at image grading scores for individual SLO wavelengths (Table 2), there is a correlation between the image quality and total cataract score for both $590 \mathrm{~nm}(\mathrm{p}=0.04)$ and $670 \mathrm{~nm}(\mathrm{p}=0.03)$ which is enhanced by removing the posterior subcapsular cataract scores $(p=0.01$ for $590 \mathrm{~nm}$ and $p=0.005$ for $670 \mathrm{~nm})$. This correlation is not apparent at $830 \mathrm{~nm}$.

\section{QUANTITATIVE DATA}

Contrast between first order retinal arterioles as they emerge from the disc and adjacent areas of background retina was calculated and expressed as a ratio of the mean grey levels within the two areas. Similar calculations were carried out for the accompanying retinal venule nearby. For all images from a single patient the same portion of vessel and background was used to allow comparison. Contrast values for retinal arterioles are shown in Table 5 and for retinal venules in Table 6. Given that these areas were of approximately 1000 pixels in size, calculation of $95 \%$
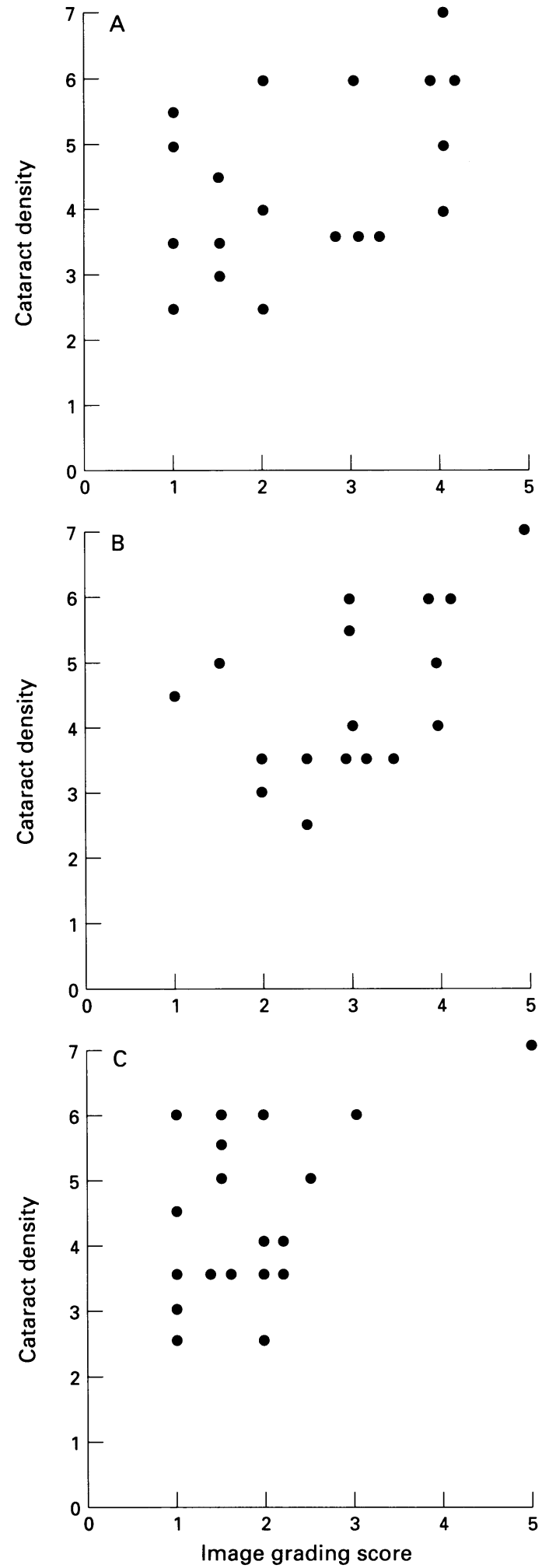

Figure 4 Scatter diagram of cataract type versus images grading score for indirect ophthalmoscopy $(A)$, fundus photography (B), and scanning laser ophthalmoscopy (C). Cataract scores are the sums of the cortical and nuclear cataract grades for each patient.

confidence limits for the grey levels shows a significant difference between all vessels and the adjacent background with the exception of one value (patient 17, $590 \mathrm{~nm}$ confocal image, Table 5). Contrast values are plotted on scatter diagrams for retinal arterioles (Fig 5) and retinal venules (Fig 6).

In general, the contrast achieved for a given vessel is significantly greater for any SLO imaging wavelength, with or without confocal 
Table 5 Contrast measurements of grey levels of peripapillary vessels compared with those of adjacent background fundus for digitised fundus photographs and scanning laser ophthalmoscopy images - arteriole to background contrast

\begin{tabular}{|c|c|c|c|c|c|c|c|}
\hline \multirow{3}{*}{$\begin{array}{l}\text { Patient } \\
\text { No }\end{array}$} & \multirow[b]{3}{*}{ Photo } & \multicolumn{6}{|c|}{ Scanning laser ophthalmoscopy } \\
\hline & & \multicolumn{2}{|l|}{$590 \mathrm{~nm}$} & \multicolumn{2}{|l|}{$670 \mathrm{~nm}$} & \multicolumn{2}{|l|}{$830 \mathrm{~nm}$} \\
\hline & & Confocal & Direct & Confocal & Direct & Confocal & Direct \\
\hline 1 & 1.037 & $1 \cdot 36$ & $1 \cdot 64$ & $1 \cdot 70$ & 1.73 & 1.88 & $1 \cdot 52$ \\
\hline 2 & 1.025 & $4 \cdot 19$ & 2.08 & $2 \cdot 18$ & $1 \cdot 75$ & 1.47 & $1 \cdot 20$ \\
\hline 3 & 0.994 & $2 \cdot 61$ & $1 \cdot 39$ & $1 \cdot 34$ & $1 \cdot 18$ & $1 \cdot 21$ & $1 \cdot 15$ \\
\hline 4 & 1.004 & N/A & 1.62 & N/A & 1.47 & N/A & 1.51 \\
\hline 5 & 0.965 & 3.08 & 3.85 & 1.50 & 1.47 & 1.90 & 1.48 \\
\hline 6 & 1.054 & $1 \cdot 82$ & 1.59 & 1.45 & $1 \cdot 20$ & 1.56 & 1.46 \\
\hline 7 & N/A & N/A & N/A & N/A & N/A & N/A & N/A \\
\hline 8 & $1 \cdot 185$ & $1 \cdot 77$ & N/A & $1 \cdot 77$ & N/A & 1.42 & N/A \\
\hline 9 & $1 \cdot 208$ & $13 \cdot 79$ & $3 \cdot 36$ & 1.29 & 0.95 & 1.79 & $1 \cdot 41$ \\
\hline 10 & 1.047 & 3.56 & $1 \cdot 10$ & $1 \cdot 12$ & $1 \cdot 17$ & 1.34 & $1 \cdot 10$ \\
\hline 11 & $1 \cdot 152$ & 1.53 & 1.81 & 1.27 & $1 \cdot 19$ & 1.59 & $1 \cdot 34$ \\
\hline 12 & 0.956 & N/A & $4 \cdot 36$ & N/A & 1.89 & N/A & $1 \cdot 18$ \\
\hline 13 & 1.029 & N/A & 1.63 & N/A & $1 \cdot 19$ & $1 \cdot 19$ & $1 \cdot 10$ \\
\hline 14 & $1 \cdot 140$ & $2 \cdot 49$ & 1.72 & 1.49 & 1.06 & $1 \cdot 34$ & 1.25 \\
\hline 15 & 0.931 & N/A & N/A & N/A & 1.43 & N/A & $1 \cdot 26$ \\
\hline 16 & $1 \cdot 108$ & N/A & $4 \cdot 87$ & $2 \cdot 61$ & 1.44 & 1.74 & 1.49 \\
\hline 17 & N/A & N/A & 1.90 & $2 \cdot 30$ & 1.62 & 1.84 & 1.85 \\
\hline 18 & $1 \cdot 159$ & 3.65 & $2 \cdot 56$ & 1.74 & 1.47 & $2 \cdot 27$ & 1.56 \\
\hline 19 & N/A & 1.89 & $2 \cdot 22$ & 0.50 & $1 \cdot 68$ & 1.41 & $1 \cdot 29$ \\
\hline
\end{tabular}

All contrast values $\mathrm{p}<0.05$.

Table 6 Contrast measurements of grey levels of peripapillary vessels compared with those of adjacent background fundus for digitised fundus photographs and scanning laser ophthalmoscopy images - venule to background contrast

\begin{tabular}{|c|c|c|c|c|c|c|c|}
\hline \multirow{3}{*}{$\begin{array}{l}\text { Patient } \\
\text { No }\end{array}$} & \multirow{3}{*}{ Photo } & \multicolumn{6}{|c|}{ Scanning laser ophthalmoscopy } \\
\hline & & \multicolumn{2}{|l|}{$590 \mathrm{~nm}$} & \multicolumn{2}{|l|}{$670 \mathrm{~nm}$} & \multicolumn{2}{|l|}{$830 \mathrm{~nm}$} \\
\hline & & Confocal & Direct & Confocal & Direct & Confocal & Direct \\
\hline 1 & $1 \cdot 256$ & $3 \cdot 20$ & $2 \cdot 26$ & 1.38 & 1.49 & 1.58 & 1.35 \\
\hline 2 & 1.043 & 6.93 & $4 \cdot 34$ & $5 \cdot 15$ & 1.82 & 1.58 & 1.33 \\
\hline 3 & $1 \cdot 204$ & $10 \cdot 84$ & $2 \cdot 87$ & 3.00 & $1 \cdot 20$ & 1.35 & $1 \cdot 15$ \\
\hline 4 & 0.855 & N/A & $7 \cdot 32$ & N/A & 1.87 & N/A & 2.04 \\
\hline 5 & $1 \cdot 200$ & $9 \cdot 81$ & 4.67 & $2 \cdot 29$ & $2 \cdot 60$ & $2 \cdot 75$ & 1.94 \\
\hline 6 & $1 \cdot 124$ & 9.97 & 4.67 & 2.06 & $1 \cdot 48$ & $2 \cdot 30$ & 1.45 \\
\hline 7 & N/A & $\mathrm{N} / \mathrm{A}$ & N/A & N/A & N/A & N/A & N/A \\
\hline 8 & $1 \cdot 264$ & $7 \cdot 26$ & N/A & $2 \cdot 29$ & N/A & $2 \cdot 18$ & N/A \\
\hline 9 & $1 \cdot 201$ & $17 \cdot 22$ & 3.59 & 1.51 & 1.35 & 1.70 & 1.64 \\
\hline 10 & 1.091 & 6.29 & $2 \cdot 14$ & 1.48 & $1 \cdot 28$ & 1.44 & 1.23 \\
\hline 11 & $1 \cdot 320$ & $7 \cdot 69$ & 8.45 & 1.90 & 1.49 & 1.88 & 1.63 \\
\hline 12 & 1.032 & N/A & 3.33 & N/A & 1.65 & N/A & $1 \cdot 12$ \\
\hline 13 & 1.035 & N/A & $3 \cdot 15$ & N/A & 1.50 & 1.64 & 1.25 \\
\hline 14 & $1 \cdot 255$ & $17 \cdot 30$ & $3 \cdot 32$ & 1.73 & 1.75 & 1.59 & 1.50 \\
\hline 15 & $1 \cdot 116$ & N/A & N/A & N/A & 1.33 & N/A & 1.45 \\
\hline 16 & 0.965 & N/A & $4 \cdot 62$ & 3.52 & 1.73 & $2 \cdot 28$ & 1.65 \\
\hline 17 & N/A & N/A & $1.73^{\star}$ & $2 \cdot 11$ & 1.64 & 1.72 & 1.31 \\
\hline 18 & $1 \cdot 272$ & $10 \cdot 58$ & $7 \cdot 77$ & $2 \cdot 23$ & 1.85 & 2.98 & 1.76 \\
\hline 19 & N/A & $8 \cdot 60$ & $4 \cdot 23$ & $5 \cdot 22$ & 1.67 & 1.66 & 1.32 \\
\hline
\end{tabular}

All contrast values except those marked $\left({ }^{\star}\right) \mathrm{p}<0.05$.

aperture, when compared with digitised fundus photographs as determined by the Wilcoxon matched pairs analysis. A typical digitised fundus image and accompanying SLO images are shown in Figure 7. Greater vessel contrast is apparent when a confocal aperture is used compared with no aperture $(p<0.001$ for arterioles and venules). Furthermore, vessel contrast is significantly greater when SLO images are obtained at $590 \mathrm{~nm}$ compared with $670 \mathrm{~nm}$ or $830 \mathrm{~nm}$ whether they be confocal or non-confocal images ( $p<0.005$ for all data subsets). There may be a trend towards slightly improved vessel contrast at $670 \mathrm{~nm}$ compared with 830 $\mathrm{nm}$ but this only achieves significance for retinal venules imaged without a confocal aperture $(p=0.02)$.

\section{Discussion}

This study has made use of subjective clinical grading systems for the assessment of cataract ${ }^{9}$ and the degree of blurring of the optic disc and retinal vasculature. ${ }^{10}$ The latter system was deemed appropriate as it is readily applied to any condition which obscures the fundus view, although it is commonly used for patients with posterior uveitis. Clinical grading systems are inherently subjective and thus subject to interpreter variation which may render them insensitive to real changes in the subjects studied. In the present study observer variation was considered and measured. Interobserver variation for the grading of cataract type was good, as was that measured for the interpretation of fundus images by all three methods. Intraobserver variation for observer one was measured by repeat grading of SLO images and was also found to be acceptable.

To allow for variations in image quality caused by minor patient movement or optical misalignment it was felt that the best of four fundus photographs and the best of two SLO images at each setting should be used for subsequent grading and image analysis.

Qualitative data from the results of grading of optic disc clarity suggest a number of points. Firstly, the image quality achieved with the SLO is evidently superior to that achieved with the 35 degree fundus camera used in this study. Secondly, compared with the image quality of indirect ophthalmoscopy, the SLO appears to show more fine fundus detail. This may be attributable to the disparity in field of view of these methods since the SLO has a narrower field and might therefore have an improved resolution.

Each of the imaging methods has its drawbacks. Indirect ophthalmoscopy provides a wide field of view but with lesser magnification than the other methods. Furthermore the resultant image is unrecordable. Fundus photography is the mainstay of permanent ophthalmic fundus recording but may not result in acceptable image quality, presumably as a result of increased light scatter in cataract patients. The SLO uses a smaller field of view and provides a monochromatic image which requires some degree of familiarity for accurate interpretation, particularly with infrared wavelengths. With the SLO the optic disc has an unusually bright optic cup and features of the fundus such as chorioretinal pigment abnormalities may appear pronounced. ${ }^{4}$ The SLO is relatively non-portable and is also more expensive.

The question of optimum SLO wavelength for imaging is not entirely clear. For the majority of patients in the study, all of whom had sufficient cataract to warrant surgery, images at $590 \mathrm{~nm}$ yielded the best grading scores. This is to be expected since the resolution of the SLO decreases for an increase in wavelength ${ }^{12}$ and, moreover, at $590 \mathrm{~nm}$ the absorbance of haemoglobin and hence the contrast of retinal vessels is many times greater compared with $670 \mathrm{~nm}$ or $830 \mathrm{~nm} .{ }^{13}$ However, there was a group of patients whose images at $590 \mathrm{~nm}$ were unrecordable as no reflected signal could be detected. In these patients $830 \mathrm{~nm}$ appeared to offer a satisfactory view of the fundus in many cases. It was also found that the gain settings for the SLO were invariably 


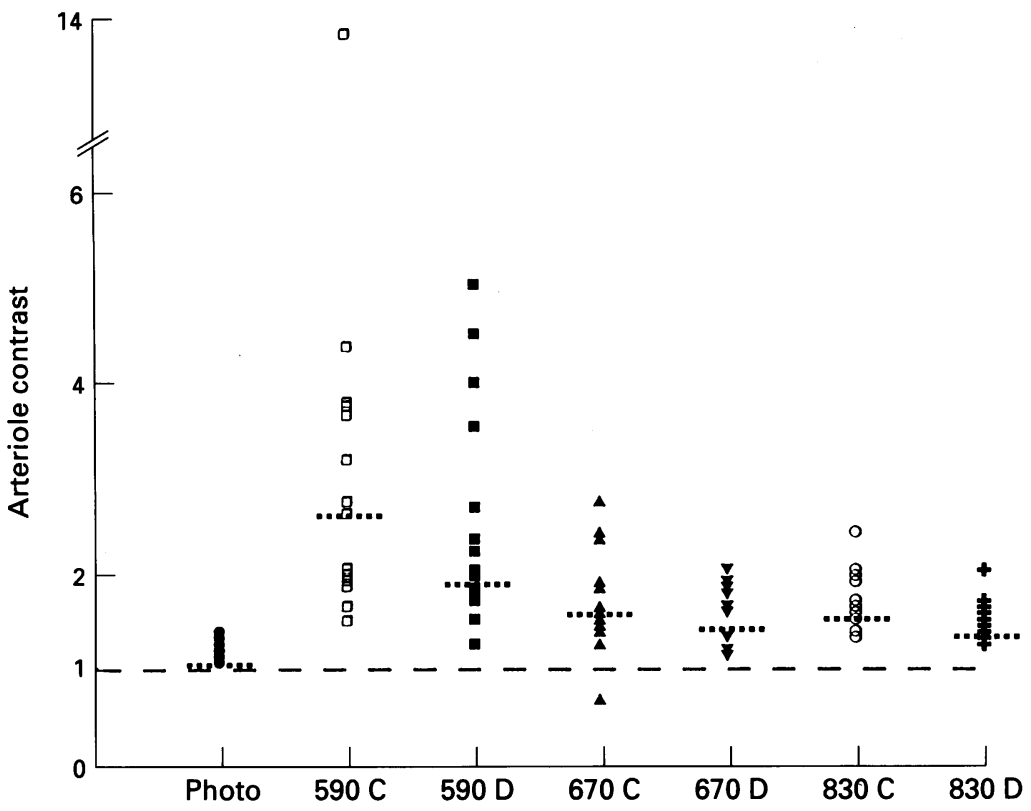

Figure 5 Scatter diagram of arteriole/background contrast ratios for indirect ophthalmoscopy, fundus photography, and scanning laser ophthalmoscopy (SLO). Median values for each subset are shown by the dotted lines. Note no data for indirect ophthalmoscopy can be recorded. Numbers on the $\mathrm{x}$ axis refer to SLO imaging wavelength and ' $C$ ' and ' $D$ ' refer to confocal and direct (non-confocal) respectively - for example, $590 \mathrm{C}$ stands for images recorded using $590 \mathrm{~nm}$ laser and confocal aperture.

highèr for $590 \mathrm{~nm}$ than $830 \mathrm{~nm}$, usually with $670 \mathrm{~nm}$ in between. Low signal returns at $590 \mathrm{~nm}$ may be due to the increased optical density of the lens at lower wavelengths, a feature of the normal lens which is exaggerated by aging ${ }^{7415}$ and this may be more pronounced in patients with cataract. In addition, the detector used in this version of the SLO has a peak sensitivity in the infrared range.

In general terms while one might expect the SLO to improve fundus image quality by illuminating the fundus through a small, clear portion of the crystalline lens, the principle of

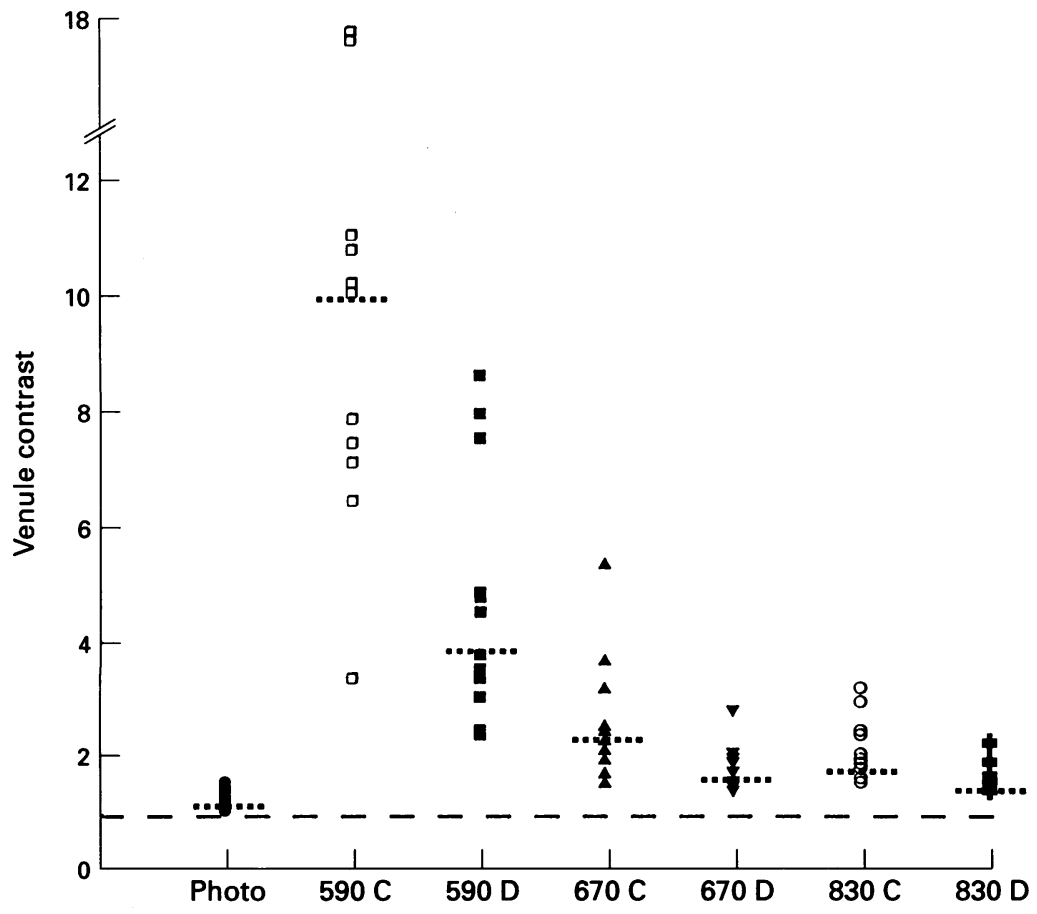

Figure 6 Scatter diagram of venule/background contrast ratios for indirect ophthalmoscopy, fundus photography, and scanning laser ophthalmoscopy. Median values for each group shown by dotted lines. Notes on axis labelling are similar to Figure 5. confocal optics offers an additional way to avoid light scattering by the lens and resultant degradation of the received image. ${ }^{16} \mathrm{With}$ this technique the SLO has the ability to create an optical sectioning or tomographic effect and so filter out light scattered from different layers, such as the lens, which would act to reduce image contrast. The $400 \mu \mathrm{m}$ confocal aperture used in this study creates an optical section thickness of $2600 \mu \mathrm{m}$ for $830 \mathrm{~nm}$ light $^{12}$ and this is not expected to vary significantly for the other wavelengths employed. Results comparing the confocal and direct (non-confocal) images show that while vessel contrast is increased significantly the clinical grading scores show no significant improvement. This is not remarkable since there were six patients in whom the use of the confocal aperture caused increased difficulty in achieving a recordable image particularly at $590 \mathrm{~nm}$, thus reducing the statistical power. In general, therefore, a confocal aperture may allow improved image quality in some patients but at the expense of the intensity of the received image.

Correlation of image quality grading with cataract type in this study should be interpreted cautiously owing to the small number of patients and the preponderance of mixed cataract classifications graded by the LOCS II system. ${ }^{9}$ Rank correlation with a summated score of cataract degree showed that indirect ophthalmoscopy images were related somewhat to the score awarded to each cataract by the observers. A significant correlation with cataract density could be achieved for indirect ophthalmoscopy and fundus photography if only scores for cortical and nuclear cataract were used. It may be that while posterior subcapsular cataract is responsible for significant functional visual loss, it contributes less to the image degradation seen when imaging in patients with a dilated pupil. The SLO showed no correlation with cataract grading scores and it is worthwhile pointing out that some patients adjudged to have high cataract density scores still had good fundus image quality scores (Fig 2). The suggestion that the SLO is best suited to a particular cataract type should be the subject of further investigation on a larger group of patients.

Quantitative data from digitised images serve to back up the qualitative data already discussed. The use of a ratio of vessel to background fundus as a measure of contrast is simple and applicable to these data since the baseline for both fundus photographs and SLO images was zero and no offset existed. The method of image capture of the fundus photographs using red free light to allow digital processing was similar to that used in other studies $^{17} 18$ and leads to minimal loss of contrast of retinal features. For those patients in whom images are obtainable, significant improvement in vessel contrast is achieved by using the SLO at any of the wavelengths tested compared with digitised fundus photographs. It appears that a striking contrast is achieved at $590 \mathrm{~nm}$ for both arterioles and venules which may be explained by selective use of a wavelength at which haemoglobin is optically dense. 


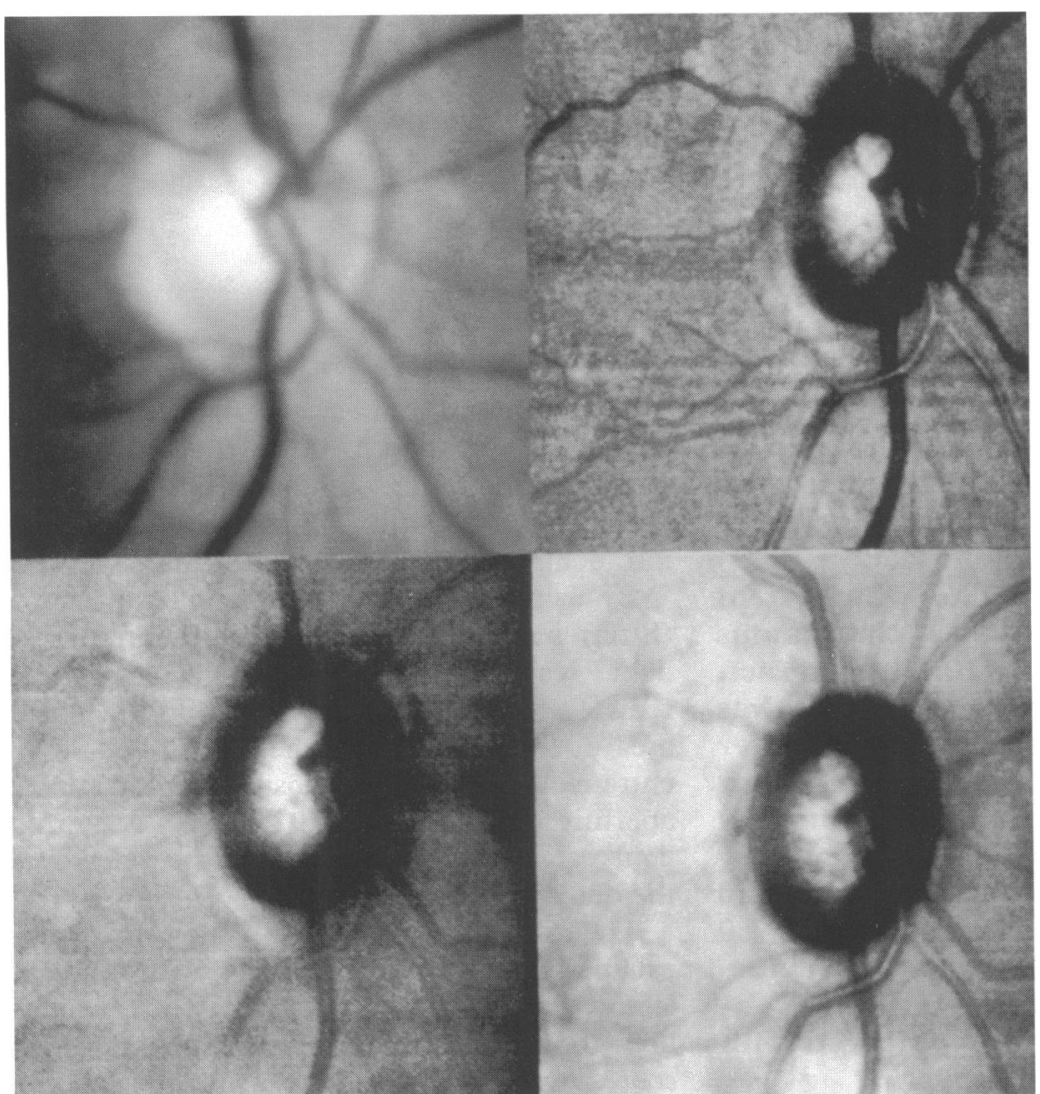

Figure 7 Comparison of digitised colour fundus photograph (top left) with scanning laser ophthalmoscopy images: $590 \mathrm{~nm}$ confocal image (top right), $670 \mathrm{~nm}$ confocal image (bottom left) and $830 \mathrm{~nm}$ confocal image (bottom right). Vessel contrast is greatest at $590 \mathrm{~nm}$. Note colour fundus photograph is rendered monochrome during digitisation process. the expense of overall image brightness and its usefulness may vary from patient to patient. The type of cataract is which SLO imaging offers most advantage is somewhat unclear and requires further study.

The authors are grateful to their sponsors for assistance with this project. Mr Kirkpatrick was supported by an ACTR fellowship from the Scottish Home and Health Department. Dr Manivannan and the image processing work station are funded by Scotia Pharmaceuticals. The scanning laser ophthalmoscope was built from funds provided by the Wellcome Trust.

The authors are grateful to Mr R Hutcheon for photographic assistance and to the technical staff of the Department of Biomedical Physics.

1 Scheider A, Nasemann JE, Lund OE. Fluorescein and indocyanine green angiographies of central serous choroidopathy by scanning laser ophthalmoscopy. Am $\mathcal{J}$ Ophthalmol 1993; 115: 50-6.

2 Wolf S, Jung F, Kiesewetter H, Körber N, Reim M. Video fluorescein angiography: method and clinical application. Graefes Arch Clin Exp Ophthalmol 1989; 227: 145-51.

3 Wolf S, Arend O, Reim M. Measurement of retinal haemodynamics with scanning laser ophthalmoscopy: reference values and variation. Surv Ophthalmol 1994; 38 (suppl): values and 100 .

4 Manivannan A, Kirkpatrick JNP, Sharp PF, Forrester JV. Clinical investigation of an infrared digital scanning laser ophthalmoscope. Br f Ophthalmol 1994; 78: 84-90.

5 Bartsch DU, Intaglietta M, Bille JF, Dreher AW, Gharib M, Freeman WR. Confocal laser tomographic analysis of the retina in eyes with macular hole formation and other focal macular diseases. Am $\mathcal{F}$ Ophthalmol 1989; 108: 277-87.

6 Burk ROW, Rohrschneider K, Takamoto T, Volcker HE, Schwartz B. Laser scanning tomography and stereophotogrammetry in three-dimensional optic disc analysis. Graefes Arch Clin Exp Ophthalmol 1993; 231: 193-8.

7 Mellerio J. Yellowing of the human lens: nuclear and cortical contributions. Vis Res 1987; 27: 1581-7.

8 Werner JS. Development of scotopic sensitivity and the absorption spectrum of the human ocular media. f Opt Soc Am 1982; 72: 247-58.

9 Chylack LT Jr, Leske MC, McCarthy D, Khu P, Kashiwag T, Sperduto R. Lens opacities classification system II (LOCS II). Arch Ophthalmol 1989; 107: 991-7.

10 Nussenblatt RB, Palestine AG, Chan CC, Roberge F. Standardization of vitreal inflammatory activity in intermediate and posterior uveitis. Ophthalmology 1985; 92 mediate

Thus the reflected signal from vessel areas is extremely low compared with background fundus. Vessel contrast is also significantly improved with the use of a confocal aperture, a finding which was not significant when analysing the qualitative data.

In conclusion, the SLO appears to offer an alternative to conventional imaging in examination of the fundi of cataract patients. For many patients the contrast of retinal features is subjectively and objectively improved compared with fundus photography. The optimum wavelength for SLO imaging from those tested appears to be $590 \mathrm{~nm}$ but because of low reflectance in some patients at this wavelength, useful data can be achieved at higher, particularly infrared, wavelengths. A confocal aperture will improve contrast but this may be at 